\title{
58. DETERMINATION OF THE FORM OF THE OORT COMETARY CLOUD AS THE HILL SURFACE IN THE GALACTIC FIELD
}

\author{
V. A. ANTONOV and I. N. LATYSHEV \\ Astronomical Observatory, Leningrad University, Leningrad, U.S.S.R.
}

\begin{abstract}
An estimate is made of the maximum range over which the motion of a particle about the Sun and in the galactic field is stable in Hill's sense. An equation fitting the Hill surface is found, with allowance made for the fact that the galactic potential differs from that of a point mass. The volume enclosed by the surface is 3.35 cubic parsecs, the greatest distance from the Sun being 1.42 parsecs, this being along the line to the centre of the Galaxy.
\end{abstract}

It is well known that the motions of the planets of the solar system are not affected to any noticeable extent by stellar perturbations. On the other hand, it is not satisfactory to regard the solar system as isolated when one is studying the motion of a comet whose orbit has a semimajor axis of $2000 \mathrm{AU}$. What would happen if the orbit of the comet (or other particle) were extended even further? It is inevitable that the solar and galactic gravitational fields of force would become comparable in their effect on the particle. The orbit would lose all semblance to a Keplerian ellipse, and many of the conventional conceptions of celestial mechanics would be invalid. In such cases the classical methods of celestial mechanics must be complemented with other methods which have been used with various degrees of success in the solution of problems of stellar dynamics.

In this paper we consider a problem in which the particle would make many revolutions about the Sun at the greatest possible distance. The size of an appropriate surface was estimated by Chebotarev $(1963,1964,1965,1966)$, who proceeded from the assumption that all the mass of the Galaxy is concentrated at its centre. In actuality, the attracting material is distributed over an extended volume, and the potential of the real galactic field clearly differs from that of a point mass. On the average, a star can be expected to pass close enough to the Sun to have a significant influence once every $10^{5} \mathrm{yr}$ or so. Accordingly, a particle with a revolution period longer than this would respond to the smoothed gravitational field of several passing stars, rather than to their individual attractions. For such a particle the attracting matter is, as it were, spread over space in a continuous manner.

The galactic orbit of the Sun is taken to be almost exactly circular. Since the mass of the Sun is small compared to that of the Galaxy it is natural to expect that the dimensions of Hill's surface would be small compared to the distance $R_{0}$ from the Sun to the galactic centre.

We shall proceed now to the analytical derivation of Hill's surface. A rotating heliocentric system of coordinates is defined such that the $x$-axis is directed away from the galactic centre, the $y$-axis in the direction of galactic rotation and the $z$-axis towards the north galactic pole. The Jacobi integral in this system of coordinates is 


$$
\begin{aligned}
W+\frac{\dot{x}^{2}+\dot{y}^{2}+\dot{z}^{2}}{2}-\frac{\omega^{2}}{2}\left[\left(R_{0}+\right.\right. & \left.x)^{2}+y^{2}\right] \\
& -u(x, y, z)-\frac{f M_{\odot}}{\sqrt{x^{2}+y^{2}+z^{2}}}=0
\end{aligned}
$$

where $x, y, z$ are the coordinates of the particle, $\omega$ is the angular velocity of the coordinate system (i.e., the angular velocity of the Sun in the Galaxy), $f$ is the constant of gravitation, $M_{\odot}$ the solar mass, $u$ the galactic potential and $W$ the constant of integration.

If we take $\dot{x}=\dot{y}=\dot{z}=0$, we obtain a family of zero velocity surfaces with parameter $W$ :

$$
W=\frac{\omega^{2}}{2}\left[\left(R_{0}+x\right)^{2}+y^{2}\right]+u(x, y, z)+\frac{f M_{\odot}}{\sqrt{x^{2}+y^{2}} \overline{\overline{1}+z^{2}}} .
$$

It is clear that a particle whose motion is governed by a Jacobi integral with a given value of $W$ is not able to pass through the surface determined by Equation (2) and must therefore remain consistently on one side of the surface.

We expand the galactic potential $u$ with respect to $x, y, z$, considering terms up to the second order only. This is simplified if we suppose that the Galaxy has a plane of symmetry $(z=0)$ and an axis of rotational symmetry. The distance $R$ from the axis of symmetry is given by

$$
R=\sqrt{\left(R_{0}+x\right)^{2}+y^{2}} \simeq R_{0}+x+\frac{y^{2}}{2 R_{0}} .
$$

Taking into account the solar motion,

$$
(\partial u / \partial R)_{0}=-\omega^{2} R_{0}
$$

it follows that

$$
\begin{aligned}
W^{\prime}= & \frac{1}{2}\left[\left(\frac{\partial^{2} u}{\partial R^{2}}\right)_{0}-\frac{1}{R_{0}}\left(\frac{\partial u}{\partial R}\right)_{0}\right] x^{2}+\frac{1}{2}\left(\frac{\partial^{2} u}{\partial z^{2}}\right)_{0} z^{2} \\
& +\frac{f M_{\odot}}{\sqrt{x^{2}+y^{2}+z^{2}}},
\end{aligned}
$$

where $W^{\prime}$ is a constant and the subscript 0 indicates that the derivatives are to be taken at the origin. For convenience we write Equation (5) in the form

$$
W^{\prime}=\frac{1}{2}\left(\alpha x^{2}+\gamma z^{2}\right)+\frac{f M_{\odot}}{\sqrt{x^{2}+y^{2}+z^{2}}}
$$

where $\alpha$ and $\gamma$ may easily be expressed in terms of the local kinematic parameters of the Galaxy. Specifically, we make use of the well-known relationship between Oort's parameters $A$ and $B$ and the linear velocity of galactic rotation $\Theta=\omega R$ :

$$
A=\frac{1}{2}\left(\frac{\Theta}{R}-\frac{\mathrm{d} \Theta}{\mathrm{d} R}\right), \quad B=-\frac{1}{2}\left(\frac{\Theta}{R}+\frac{\mathrm{d} \Theta}{\mathrm{d} R}\right) .
$$


Numerical values, according to Schmidt (1965), are

$$
A=15 \mathrm{~km} \mathrm{~s}^{-1} \mathrm{kpc}^{-1}, \quad B=-10 \mathrm{~km} \mathrm{~s}^{-1} \mathrm{kpc}^{-1} \text {. }
$$

Solar motion may be taken as a specific phenomenon of galactic rotation for $R=R_{0}$, the peculiar velocity of the Sun being small compared with $\Theta$. Differentiating Equation (4) and comparing it with Equation (7), we obtain

$$
\alpha=4 A(A-B)
$$

and according to Agekyan et al. (1962), $\gamma$ may be written

$$
\gamma=-C^{2}
$$

where $C=68 \mathrm{~km} \mathrm{~s}^{-1} \mathrm{kpc}^{-1}$.

It is significant that the above procedure did not require any model for the Galaxy as a whole. Indeed, we have reduced everything to consideration of the observational parameters $A, B$, and $C$ and have not had to make any assumptions about the structure of the Galaxy at great distances. For large values of $W^{\prime}$ Equation (6) gives almost spherical surfaces encircling the Sun. But our interest is in the critical Hill surface, i.e., a surface which ceases to be bounded as $W^{\prime}$ decreases further. A simple analysis shows that the critical value of $W^{\prime}$ is

$$
W^{\prime}=\frac{3}{2} f^{2 / 3} M_{\odot}^{2 / 3} \alpha^{1 / 3}
$$

For this value of $W^{\prime}$ two specific conical points, given by

$$
y=z=0, \quad x= \pm \sqrt[3]{\frac{f M_{\odot}}{\alpha}},
$$

will appear on the surface. Apart from these points the surface is smooth and resembles a triaxial ellipsoid whose major axis coincides with the $x$-axis. Numerical values for the semiaxes are

$$
\begin{aligned}
& x_{\max }=1.42 \mathrm{pc}=293 \times 10^{3} \mathrm{AU} \\
& y_{\max }=0.92 \mathrm{pc}=196 \times 10^{3} \mathrm{AU} \\
& z_{\max }=0.74 \mathrm{pc}=152 \times 10^{3} \mathrm{AU} .
\end{aligned}
$$

The triaxial form would be preserved in the case of a spherically symmetric gravitational field as well. Figure 1 shows a section by the plane $x 0 z$ of the surfaces for different values of $W^{\prime}$.

The assumption that the closed Hill surface is small compared to galactic dimensions seems to be justified. It is interesting that this is the case for our Galaxy, for the situation would be very different in the inner parts of an elliptical galaxy. Then $\omega \approx$ const, the parameter $A$ is small and the Hill surface is much larger, which indicates that a particle can return to its star after covering a distance several times the mean interstellar distance.

But in our Galaxy the volume of the critical Hill surface about the Sun is $28 \%$ of what it would be if all the stars were uniformly distributed. In absolute terms the volume 
is $3.35 \mathrm{pc}^{3}$. This estimate was obtained by means of an approximate numerical integration; despite a thorough investigation we have not been able to determine this volume analytically.

The two critical points are merely libration points in the particle-Sun-Galaxy system without the assumption of Newtonian gravitation. This modified three-body problem, like the basic problem, contains five libration points (Duboshin, 1969), but the other three points are far from the Sun and thus devoid of any physical meaning.

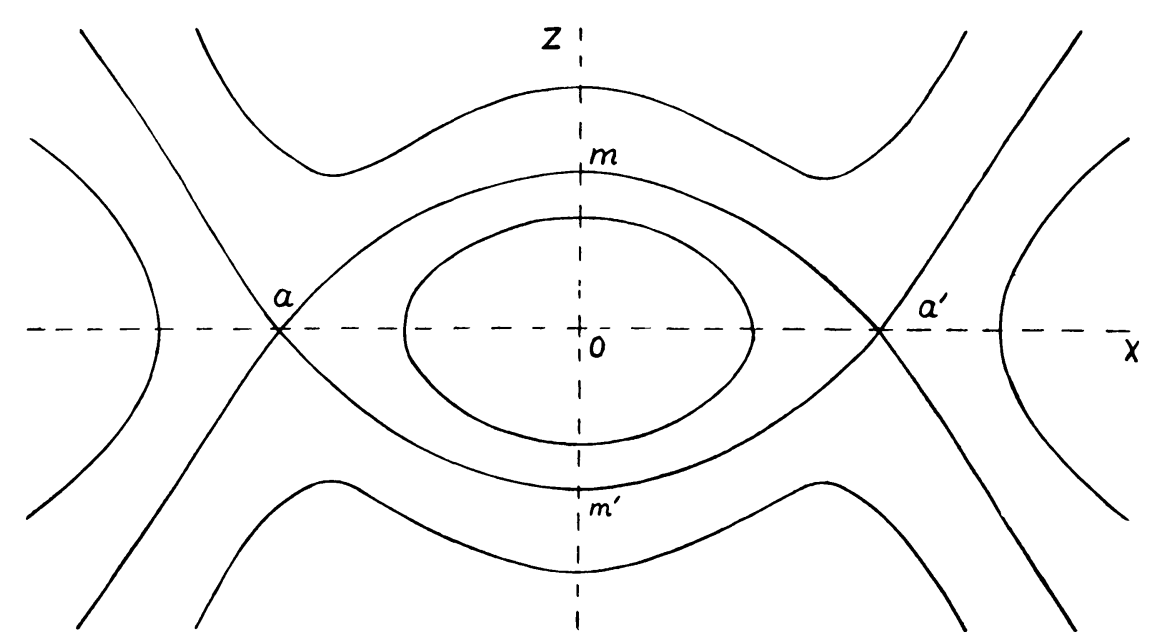

Fig. 1. A section by the plane $x 0 z$ of the surfaces for different values of $W^{\prime}$. The critical points are $a$ and $a^{\prime}$ and the critical surface $a m a^{\prime} m^{\prime}$.

In conclusion, briefly we consider some further questions:

(1) Will a particle for which the surface given by Equation (6) is not closed be certain to escape from the Sun? The problem involves the existence of other integrals of motion in addition to that of Jacobi. There is reason to believe that the meanderings of the orbit are not limited; at least, something of this kind is observed in related numerical experiments on galactic orbits of stars (Aarseth, 1966).

(2) What is the physical interpretation of the problem? Our approach is confined to considering the geometry of possible orbits and does not involve any numerical predictions concerning the density of the circumsolar cloud. Nor do we wish to state the cosmogonical importance of particles moving in extremely extended orbits. It is quite possible that our hypothetical particles bear no relation at all to comets that could be seen from the Earth. On the other hand, statements concerning a circumsolar cloud at the distance of 1 to $2 \times 10^{5} \mathrm{AU}$ can, in principle, be checked by analogy, since similar particles from other stars should, even if very rarely, enter the solar system and be detected. In the solar system, they will be easily distinguished by their highly hyperbolic orbits. Further, we may expect to find, not only comet-like, but also star-like satellites at these great distances from a particular star. Such extended 
binary systems have recently begun to interest astronomers; and there is no a priori reason why the Sun should not have a dwarf, stellar satellite.

\section{References}

Aarseth, S. J.: 1966, Nature 212, 57.

Agekyan, T. A., Vorontsov-Vel'yaminov, B. A., Gorbazkij, V. G., Deutsch, A. N., Krat, V. A., Melnikov, O. A., and Sobolev, V. V.: 1962, Kurs Astrofiziki i Zvezdnoj Astronomii, Fitmatgiz, Moscow, Vol. II, section 158.

Chebotarev, G. A.: 1963, Astron. Zh. 40, 812.

Chebotarev, G. A.: 1964, Astron. Zh. 41, 983.

Chebotarev, G. A.: 1965, Analiticheskie $i$ Chislennye Metody Nebesnoj Mekhaniki, Nauka, Moscow and Leningrad (English translation: 1967, Analytical and Numerical Methods of Celestial Mechanics, Elsevier, New York), Chapter 6.

Chebotarev, G. A.: 1966, Astron. Zh. 43, 435.

Duboshin, G. N.: 1969, Astron. Zh. 46, 1279.

Schmidt, M.: 1965, in A. Blaauw and M. Schmidt (eds.), Galactic Structure, University of Chicago Press, Chicago and London, p. 513. 